\title{
An Update on Binary Formation by Rotational Fission
}

\author{
Joel E. Tohline \\ Louisiana State University, Department of Physics and Astronomy, \\ Baton Rouge, LA 70803-4001, U.S.A. \\ Richard H. Durisen \\ Department of Astronomy, Swain West 319, Indiana University, \\ Bloomington, IN 47405, U.S.A.
}

\begin{abstract}
During the 1980s, numerical simulations showed that dynamic growth of a barlike mode in initially axisymmetric, equilibrium protostars does not lead to prompt binary formation, i.e., fission. Instead, such evolutions usually produce a dynamically stable, spinning barlike configuration. In recent years, this result has been confirmed by numerous groups using a variety of different hydrodynamical tools, and stability analyses have convincingly shown that fission does not occur in such systems because gravitational torques cause nonlinear saturation of the mode amplitude. Other possible routes to fission have been much less well scrutinized because they rely upon a detailed understanding of the structure and stability of initially nonaxisymmetric structures and/or evolutions that are driven by secular, rather than dynamic processes. Efforts are underway to examine these other fission scenarios.
\end{abstract}

\section{Relevant Results up through the 1980s}

In the context of binary star formation, "fission" is the hypothetical process by which a rotating, equilibrium protostellar core becomes unstable to the spontaneous growth of nonaxisymmetric structure which, when fully developed, causes it to break into two or more pieces. This concept dates back over 100 years to stability analyses which showed that rapidly rotating, axisymmetric fluid configurations are unstable to the growth of an ellipsoidal or barlike structure (Chandrasekhar 1969; Tassoul 1978; Durisen \& Tohline 1985). For example, dynamic instability of a barlike distortion occurs when the ratio of rotational to gravitational potential energy $T /|W| \gtrsim 0.27$. When $T /|W| \gtrsim 0.14$, axisymmetric systems can evolve to lower energy, barlike configurations in the presence of dissipative mechanisms (e.g., viscosity or gravitational radiation). In this case, evolution proceeds on a secular time scale that is long compared to the dynamic time scale. Early stability analyses were unable to determine whether these types of instabilities would ultimately lead to fission due to the difficulty of modeling nonlinear-amplitude figure distortions for realistic fluids.

In the 1980s, the development of 3D hydrodynamics codes and advances in computing technologies made it possible to study the linear and nonlinear 
development of the barlike dynamic instabilities in realistic protostar models under a variety of physical conditions. In the linear regime, there was general agreement between the results of these earliest hydrodynamical simulations and the predictions of linear theory - specifically, on the value of $T /|W|$ at which the dynamic barlike instabilities set in and on the growth rates and pattern frequencies of the unstable modes. Most importantly, as first reported by Durisen et al. (1986), the unstable barlike mode eigenfunction for differentially rotating, compressible fluids possesses an open, two-armed spiral character which is able to dynamically redistribute angular momentum via gravitational torques and thereby prevent the model from undergoing fission. Instead, the simulations generally produced a centrally located, triaxial (barlike) structure, which was spinning about its shortest axis and surrounded by a relatively low-mass disk (or ring) of high specific angular momentum debris. We closed out the 1980s knowing that the dynamic instability of barlike modes, which can arise naturally in rapidly rotating, axisymmetric protostars (see Bate 1998 for a recent example), does not lead directly to the formation of binary stars. Many researchers concluded from this that the "fission hypothesis" was dead.

However, because the appropriate numerical tools were not yet available, we learned little in the 80s about the nonlinear development of secular instabilities or about the structure and stability of rapidly rotating, equilibrium configurations that have steady-state, nonaxisymmetric structures. The latter is especially important because, as Lebovitz $(1987 \mathrm{a}, \mathrm{b})$ hypothesized, binary fission may result from the slow contraction of such configurations.

\section{Results from the Past Decade}

\subsection{Confirmation of Earlier Dynamic Barlike Mode Results}

Over the past decade, a number of different groups using a variety of hydrodynamics codes have studied the onset and development of the dynamic barlike instability in initially axisymmetric fluid objects, both in the context of star formation and in the context of the late stages of stellar evolution (see Imamura, Durisen \& Pickett 2000, Cazes \& Tohline 2000, Durisen et al. 2000, New, Centrella \& Tohline 2000, Brown 2000, Shibata, Baumgarte \& Shapiro 2000, and references therein). Most importantly in the context of this review, these recent simulations have confirmed the following: (1) The dynamical barmode instability does not lead to prompt binary fission. (2) The instability usually leads to the formation of a dynamically stable, barlike structure that is spinning about its shortest axis and has nontrivial internal flows.

As Imamura et al. (2000) have most clearly demonstrated, prompt binary fission is prevented because the barlike mode saturates once the time scale for angular momentum transport by gravitational torques becomes as short as the growth time for the mode. When the barlike eigenfunction computed by linear analysis is artificially pushed to a large absolute amplitude, the configuration takes on the appearance of a common-envelope binary with well-defined circulation patterns isolated about each of the two off-axis density enhancements. But, in hydrodynamical simulations, the barlike mode amplitude saturates at a value well below what is required to realize this binary structure. The relative prominence of the final barlike structure depends on the degree of compressibility of 
the gas, on the distribution of angular momentum in the initial axisymmetric cloud, and on the extent to which shock dissipation is permitted to heat the gas during an evolution (Durisen et al. 2000). Although the bars that form appear to be dynamically stable (Cazes \& Tohline 2000), it seems clear that they must continue to evolve on a secular time scale as processes, such as ongoing dissipation through shocks, force slow changes in their overall structure. It is not yet clear how long the bar will live in various realistic physical systems.

\subsection{Investigation of Lebovitz's Hypothesis}

The evolutionary scenario discussed above begins from a dynamically unstable initial axisymmetric object. Lebovitz $(1987 \mathrm{a}, \mathrm{b})$ suggested instead that binary fission occurs via the slow, quasi-static contraction of inviscid protostars that have initially triaxial structures. This has been more difficult to examine realistically because, until recently, we have not had the tools to construct steady-state, nonaxisymmetric models, let alone follow their slow secular evolution.

Andalib (1998; see also Tohline, Cazes \& Cohl 1999) has now developed an SCF technique that permits the construction of differentially rotating, nonaxisymmetric equilibrium structures with moderately compressible equations of state (EOS). Models along some of Andalib's sequences deform smoothly from an elliptical, to a dumbbell, to a binary state. Shu (these proceedings; see also Shu et al. 2000) has also demonstrated that a variety of nonaxisymmetric equilibrium models of isothermal clouds can be constructed analytically. So far, in both cases, the models are infinitesimally thin; but they suggest that 3D nonaxisymmetric models with realistic EOS's may be feasible.

With Lebovitz's hypothesis specifically in mind, Cazes (1999) recently followed the slow contraction of the dynamically stable, steady-state, barlike structure that resulted from an earlier 3D barlike mode simulation. In order to induce the bar to contract, Cazes simulated cooling by forcing the polytropic constant $K$ in his EOS to decrease linearly with time according to $K=K_{o}\left(1-t / t_{\text {cool }}\right)$, with $t_{\text {cool }}=4 P_{\text {pat }}$, where $P_{\text {pat }}$ is the initial pattern rotation period of the barlike shape. This cooling was slow enough that the configuration remained close to virial equilibrium throughout. As predicted by Lebovitz, the model became more and more elongated as it cooled. Then, after $K$ had decreased to approximately half of its original value, the model began to oscillate dynamically between a centrally condensed, barlike state and a distinctly dumbbell state. In its dumbbell state, the model presented a pair of clearly defined off-axis density maxima, and a velocity field that showed circulation about each density maximum.

As described by Cazes (1999), the bar had reached a point in its evolution where there were two equally plausible equilibrium configurations into which it was permitted to settle and that, upon further cooling, the system was likely to evolve toward a common-envelope binary state (Tohline et al. 1999). On the other hand, the dumbbell structure described by Cazes resembles in many respects a highly nonlinear-amplitude realization of the standard barlike eigenfunction (Imamura et al. 2000). Unfortunately, due to computational constraints, Cazes was unable to follow his cooling evolution further; and, when cooling was stopped, the model settled into a centrally condensed, rather than a binary configuration. So it is debatable whether Cazes' model was actually progressing along a route to fission. Nevertheless, his simulation provides the most tantaliz- 
ing evidence to date that the Lebovitz fission hypothesis may be correct under some conditions.

\section{Work to Be Done}

With regard to the dynamic barlike instability, the ultimate fate of the bar and its surrounding disk of debris is still not clear. To resolve this uncertainty, relevant heating and cooling processes must be treated correctly; and the dynamics of the disk debris must be followed for many dynamical times. Given that tools are being developed to construct equilibrium models of nonaxisymmetric objects, we may soon be in a position to evaluate critically the binary fission hypothesis proposed by Lebovitz. Efforts also need to be made to study the nonlinear development of the secular barlike instabilities.

\section{References}

Andalib, S. W. 1998, Ph.D. Dissertation, Louisiana State University

Bate, M. R. 1998, ApJL, 508, L95

Brown, D. 2000, //arxiv.org/abs/gr-qc/0004002

Cazes, J. E. 1999, Ph.D. Dissertation, Louisiana State University

Cazes, J. E., \& Tohline, J. E. 2000, ApJ, 532, 1051

Chandrasekhar, S. 1969, Ellipsoidal Figures of Equilibrium (New Haven: Yale Univ. Press)

Durisen, R. H., Gingold, R. A., Tohline, J. E., \& Boss, A. P. 1986, ApJ, 305, 281

Durisen, R. H., Pickett, B. K., Bate, M. R., Imamura, J. N., Brandl, A., \& Sterzik, M. F. 2000, in IAU Symp. 200, Poster Proceedings, ed. B. Reipurth \& H. Zinnecker (Potsdam), 187

Durisen, R. H., \& Tohline, J. E. 1985, in Protostars \& Planets II, ed. D. C. Black \& M. S. Matthews (Tucson: Univ. Arizona Press), 534

Imamura, J. N., Durisen, R. H., \& Pickett, B. K. 2000, ApJ, 528, 946

Lebovitz, N. R. 1987a, Geophys Ap Fluid Dynamics, 38, 15

Lebovitz, N. R. 1987b, in Highlights of Astronomy Vol. 8, ed. D. McNally (Boston: Kluwer), 129

New, K. C. B., Centrella, J. M., \& Tohline, J. E. 2000, Phys. Rev. D, in press

Shu, F. H., Laughlin, G., Lizano, S., \& Galli, D. 2000, ApJ, 535, 190

Tassoul, J.-L. 1978, Theory of Rotating Stars (Princeton: Princeton Univ. Press)

Tohline, J. E., Cazes, J. E., \& Cohl, H. S. 1999, in Numerical Astrophysics, ed. S. M. Miyama, K. Tomisaka \& T. Hanawa (Boston: Kluwer), 155

Shibata, M., Baumgarte, T. W., \& Shapiro, S. L. 2000, ApJ, in press 

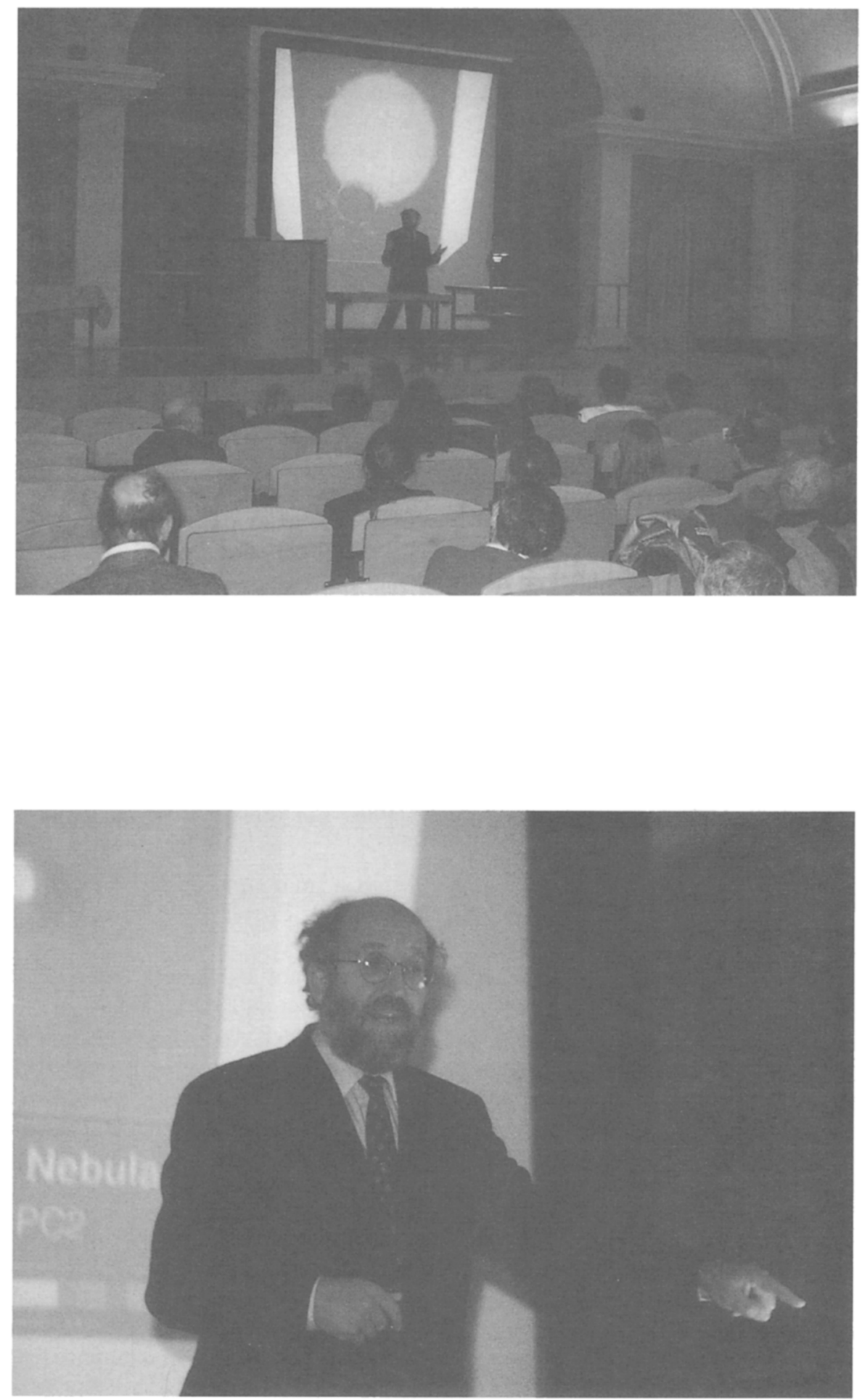

Michel Mayor during his public lecture at the University of Potsdam 